\title{
Prototipação de um recurso de comunicação alternativa para a pessoa com
}

\section{traqueostomia por câncer de laringe}

\author{
Prototyping of an alternative communication resource for the person with laryngeal cancer \\ tracheostomy
}

Creación de un prototipo de un recurso de comunicación alternativa para la persona con cáncer de laringe traqueotomía

\author{
Wagner Felipe dos Santos Neves \\ ORCID: https://orcid.org/0000-0001-9989-7273 \\ Universidade do Estado do Pará, Brasil \\ E-mail: wagnerfelipeneves@usp.br \\ Antonio Jorge Silva Correa Junior \\ ORCID: https://orcid.org/0000-0003-1665-1521 \\ Universidade de São Paulo, Brasil \\ E-mail: antoniocorreajunior@usp.br \\ Andre Lucas de Lima Dias \\ ORCID: https://orcid.org/ 0000-0002-6820-6801 \\ Universidade do Estado do Pará, Brasil \\ E-mail: diaslucasandre@gmail.com \\ Helena Megumi Sonobe \\ ORCID: https://orcid.org/0000-0003-3722-0835 \\ Universidade de São Paulo, Brasil \\ E-mail: megumi@eerp.usp.br \\ Mary Elizabeth de Santana \\ ORCID: https://orcid.org/0000-0002-3629-8932 \\ Universidade do Estado do Pará, Brasil \\ E-mail: mary.santana@uepa.br
}

\begin{abstract}
Resumo
Objetivo: realizar a prototipação de um aplicativo móvel de comunicação alternativa para pessoas com traqueostomia por câncer de laringe, fundamentada na Teoria de Necessidades Humanas Básicas de Horta. Métodos: estudo de aplicação do modelo de criação de protótipos de Pressman, com adoção do formato em papel pela adequacidade da apresentação da concepção tecnológica, com implementação das fases de definição e de desenvolvimento. Por se tratar de uma pesquisa na área da Enfermagem adotou-se a Teoria das Necessidades Humanas Básicas de Wanda Horta, focalizando-se a comunicação. Resultados: o protótipo proposto foi a intervenção diante da interrupção do processo de comunicação destes adoecidos. Considerações finais: o método de prototipação viabilizou a concepção de uma tecnologia, com o planejamento e discussão detalhada de cada uma das etapas, confirmando-se a viabilidade do desenvolvimento de um software, neste caso um aplicativo móvel, fundamentada na Teoria de Necessidades Humanas Básicas, vinculado ao Processo de Enfermagem.

Palavras-chave: Enfermagem oncológica; Aplicações da informática médica; Neoplasias laríngeas, Teoria de enfermagem; Auxiliares de comunicação para pessoas com deficiência.

Abstract

Objective: to carry out the prototyping of an alternative communication mobile application for people with laryngeal cancer tracheostomy, based on the Wanda Horta's theory of Basic Human Needs. Methods: application study of the Pressman prototype creation model, with the adoption of the paper format due to the adequacy of the presentation of the technological conception, with the implementation of the definition and development phases. As this is a research in the Nursing area, Wanda Horta's Theory of Basic Human Needs was adopted, focusing on communication. Results: the proposed prototype was the intervention in view of the interruption of the communication process of these patients. Final considerations: the prototyping method enabled the conception of a technology, with the planning and detailed discussion of each of the stages, confirming the feasibility of developing a software, in this case a mobile application, based on the Theory of Basic Human Needs linked to the Nursing Process.
\end{abstract}

Keywords: Oncology nursing; Medical informatics applications; Laryngeal neoplasms; Nursing theory; Communication aids for disabled. 


\begin{abstract}
Resumen
Objetivo: realizar el prototipado de una aplicación móvil de comunicación alternativa para personas con traqueotomía por cáncer de laringe, basada en la Teoría de Necesidades Humanas Básicas de Horta. Métodos: estudio de aplicación del modelo de creación del prototipo Pressman, con la adopción del formato papel por adecuación de la presentación de la concepción tecnológica, con la implementación de las fases de definición y desarrollo. Al tratarse de una investigación en el área de Enfermería, se adoptó la Teoría de las Necesidades Humanas Básicas de Wanda Horta, enfocada en la comunicación. Resultados: el prototipo propuesto fue la intervención ante la interrupción del proceso de comunicación de estos pacientes. Consideraciones finales: el método de prototipado permitió la concepción de una tecnología, con la planificación y discusión detallada de cada una de las etapas, confirmando la viabilidad de desarrollar un software, en este caso una aplicación móvil, basado en la Teoría de las Necesidades Humanas Básicas vinculadas a el proceso de enfermería.
\end{abstract}

Palabras clave: Enfermería oncológica; Aplicaciones de la informática médica; Neoplasias laríngeas; Teoría de enfermería; Equipos de comunicación para personas con discapacidad.

\title{
1. Introdução
}

O tratamento cirúrgico para o câncer de laringe em estágio avançado é a laringectomia total (Chaves \& Pernambuco et al., 2012), que resulta em repercussões de maior severidade, que reduzem a qualidade de vida destas pessoas, comprometendo as atividades de vida diária e, quando ocorrem complicações graves, podem levar ao óbito (Galbiatti et al., 2013) . Desta forma, o laringectomizado total apresenta alterações definitivas como afonia e a confecção de traqueostomia pela remoção de estruturas de produção do som laríngeo, assim como do fluxo aéreo definitivo via traqueostomia e alterações do olfato e paladar, em decorrência do tratamento neoadjuvante/adjuvante de radioterapia (Caldas, Facundes \& Melo, 2011). Estas alterações impactam na autoestima e em todas as dimensões da vida da pessoa e das pessoas de seu convívio, com mudanças na dinâmica familiar e social, sendo que a comunicação entre as pessoas, em geral, ainda está focalizada na verbal falada.

A capacidade de comunicação humana é uma função fundamental por permitir a expressão de pensamentos e opiniões, que possibilita entender, construir e estabelecer relacionamentos entre as pessoas e para a integração ativa nas comunidades sociais aos quais pertence (Fogle, 2013).

Após o tratamento cirúrgico, os pacientes necessitam aprender alternativas para a comunicação verbal falada, que a capacidade de produção da voz é perdida abruptamente, causando estranhamento e dificuldades em relação à afonia e à traqueostomia, tanto para os pacientes como de seus cuidadores. As possibilidades da comunicação verbal passam a ser constituídas pela Voz esofágica (VE); Voz com eletrolaringe (VEL) e Voz traqueoesofágica (VTE), além da comunicação não verbal por meio de gestos e mímica labial. Para cada uma dessas possibilidades há necessidade de intenso treinamento, trazendo desafios específicos à comunicação destes pacientes.

Para que o cuidado deste paciente seja eficaz, é importante a atenção à qualidade de vida do paciente (Othero \& Costa, 2007), que inclui o controle da doença e dos sintomas durante o seguimento ambulatorial e a reabilitação destas pessoas em relação as repercussões do tratamento cirúrgico. Os recursos de Tecnologia no processo de reabilitação possibilitam preservar as atividades diárias de vida e garantir uma melhor qualidade de vida $(\mathrm{QV})$ ao paciente oncológico (Melo Filho et al., 2013; Silveira et al., 2012). Para tanto, as alternativas para a comunicação devem ser abordadas com os pacientes e familiares no ensino pré-operatório e retomadas no pós-operatório.

Assim, os sistemas de comunicação podem ser auxiliares ou suplementares, a depender das necessidades, permitindo a escolha dos pacientes laringectomizados totais ou parciais, de acordo com seus desejos, para favorecer a sua participação social. A comunicação pode ocorrer por meio de linguagem escrita, gestos, expressões faciais, uso de pranchas de alfabeto ou símbolos pictográficos, além do uso de sistemas mais sofisticados como comunicadores de voz, computadores e tablets (Pelosi, 2011). No Brasil, em 2016, os smartphones ocuparam a primeira posição entre os dispositivos eletrônicos, mais consumidos (Deloitte Touche Tohmatsu Limited, 2016), sendo que a incorporação de tais tecnologias no âmbito dos serviços de saúde, potencializam as transformações na melhoria do processo de cuidado (Agboola et al., 2016; Juliani et.al, 2014). 
Neste contexto, a incorporação de Tecnologias de Informação em Saúde, na assistência de enfermagem aos adoecidos com traqueostomia por câncer de laringe por meio de aplicativo, poderá facilitar a expressão de suas demandas de necessidades. A proposição de um protótipo deste aplicativo, foi realizada mediante a concepção de uma tecnologia, adotandose o método de prototipação (Pressman, 2006), que assegurou o embasamento teórico e o rigor metodológico para o desenvolvimento de software deste estudo.

Este estudo objetivou realizar a prototipação de um aplicativo móvel como alternativa de comunicação para pessoas com traqueostomia por câncer de laringe, fundamentada na Teoria de Necessidades Humanas Básicas de Horta (1979).

\section{Metodologia}

Trata-se de um estudo de natureza aplicada, sendo este se trata de estudo metodológico, uma vez que seu produto advém de um trabalho rigoroso, meticuloso, que usa conhecimentos decorrentes da pesquisa ou experiência prática com vistas à produção de novos materiais, equipamentos, políticas e comportamentos (Gil, 2010).

Para a proposição do produto tecnológico, buscou-se compreender os processos da engenharia de software, da qual adotou-se o modelo teórico de criação de protótipos de Pressman (2006), o qual propõe três formas: (1) protótipo em papel ou por modelo de software; (2) protótipo de trabalho com funções pré-estabelecidas; (3) programa existente com características a ser aperfeiçoadas. Escolheu-se a forma protótipo em papel ou modelo de software, pela adequacidade da apresentação da concepção da tecnologia aos usuários.

Um protótipo é uma versão inicial de um sistema de software, usado para demonstrar conceitos, experimentar opções de projeto e descobrir mais sobre o problema e suas possíveis soluções, ainda na esfera teórica, pois trabalhou-se as fases definição e desenvolvimento do modelo de Pressman. Protótipos possibilitam, ao usuário, avaliar a eficiência de atendimento das suas necessidades pelo sistema proposto, com definição das técnicas e dos materiais para o seu desenvolvimento (Somerville, 2011). O protótipo foi desenvolvido na cidade de Belém do Pará, no período de agosto de 2019 a março de 2020.

A concepção da tecnologia de comunicação alternativa de pessoas com traqueostomia pelo câncer de laringe, foi fundamentada na Teoria de Necessidades Humanas Básicas de Wanda Horta (1979) para viabilizar a expressão das necessidades humanas básicas da pessoa com traqueostomia por câncer de laringe. Esta etapa do estudo foi realizada por uma equipe de pesquisa, composta por um graduando, orientado por duas enfermeiras doutoras e docentes, além de um enfermeiro mestre, todos com expertise na assistência e na pesquisa em enfermagem oncológica, na assistência de enfermagem às pessoas com câncer de laringe e câncer colorretal (Neves \& Santana, 2020).

Por se tratar de dados secundários, não houve a necessidade de aprovação de Comitê de Ética, também não envolveu riscos de exposição de dados do público-alvo.

\subsection{Protocolo do estudo}

O processo de desenvolvimento do protótipo foi embasado na engenharia de software de Pressman (2006), que é realizado em três fases: Fase de definição dos requisitos da aplicação, com verificação da sua viabilidade e planejamento das atividades a serem desenvolvidas; Fase de desenvolvimento da produção do software (concepção, especificação, design da interface, prototipação dentre outras); e Fase de manutenção do aplicativo quando é efetivamente utilizado pelos usuários, permitindo os feedbacks destes para a otimização do sistema. Neste estudo foram desenvolvidas as fases de definição e de desenvolvimento. 


\subsection{Fase de definição}

No planejamento das atividades, inicialmente foi determinado o percurso metodológico da criação do protótipo, no qual realizou-se uma revisão da literatura acerca das formas de comunicação alternativa da pessoa com traqueostomia por câncer de laringe, bem como as tecnologias de informação em saúde e na enfermagem e a relação da enfermagem com a informática. Após a organização dos dados coletados decidiu-se adotar a Teoria de Necessidades Humanas Básicas para fundamentar o protótipo da tecnologia, em virtude de a comunicação ser um problema potencial na vida e no cuidado da pessoa com traqueostomia por câncer de laringe. Tendo em vista que a comunicação constitui um instrumento de enfermagem e uma necessidade humana básica, a fundamentação do protótipo nesta teoria apresenta-se como um recurso norteador da consecução do Processo de Enfermagem para esta clientela, e para o embasamento científico da área, na construção de um recurso tecnológico. Sequencialmente, realizou-se a fase de concepção da tecnologia, constituída pelo processo de análise e especificação de requisitos, que posteriormente fundamentarão a programação da tecnologia.

\subsection{Fase de desenvolvimento}

Essa fase traduziu os requisitos, previamente definidos pela revisão da literatura, associados aos dados coletados e à experiência clínica dos pesquisadores sobre o processo comunicacional de pessoas com traqueostomia por câncer de laringe. Representou a ilustração das ideias e dos conceitos apresentados, nas fases anteriores do modelo de prototipação, como um produto gerado pela associação das ideias, conceitos e literatura analisada, que resultou no protótipo em papel. O desenvolvimento da interface, da identidade visual do app e do design da tecnologia apresentada, foi viabilizada com o auxílio de um design gráfico, responsável pela criação das imagens do protótipo.

\section{Resultados}

Por meio do protótipo apresentado, desenvolveu-se o protótipo de um aplicativo para a viabilização da expressão das necessidades humanas básicas da pessoa com traqueostomia por câncer de laringe. Objetivou-se o desenvolvimento de um aplicativo multiplataforma, isto é, que tivesse uma funcionalidade nos principais sistemas operacionais do mercado: IOS e Android e que pudesse estar disponível para uso offline, ocupando pouco espaço no dispositivo do usuário, para assegurar a acessibilidade e a-sua funcionalidade em qualquer smartphone, sem a necessidade de uma conexão de internet ativa.

A aplicação móvel contará com um espaço pessoal, em que o usuário adicionará informações como sua identificação, seu peso e uma foto, para promover a individualização na utilização do app, e principalmente, a interação com os desenvolvedores da tecnologia, por meio do envio de sugestões para o aprimoramento do aplicativo, como na Figura 1. 
Figura 1 - Representação da tela inicial do App com as grandes categorias. Belém, PA, Brasil, 2021.

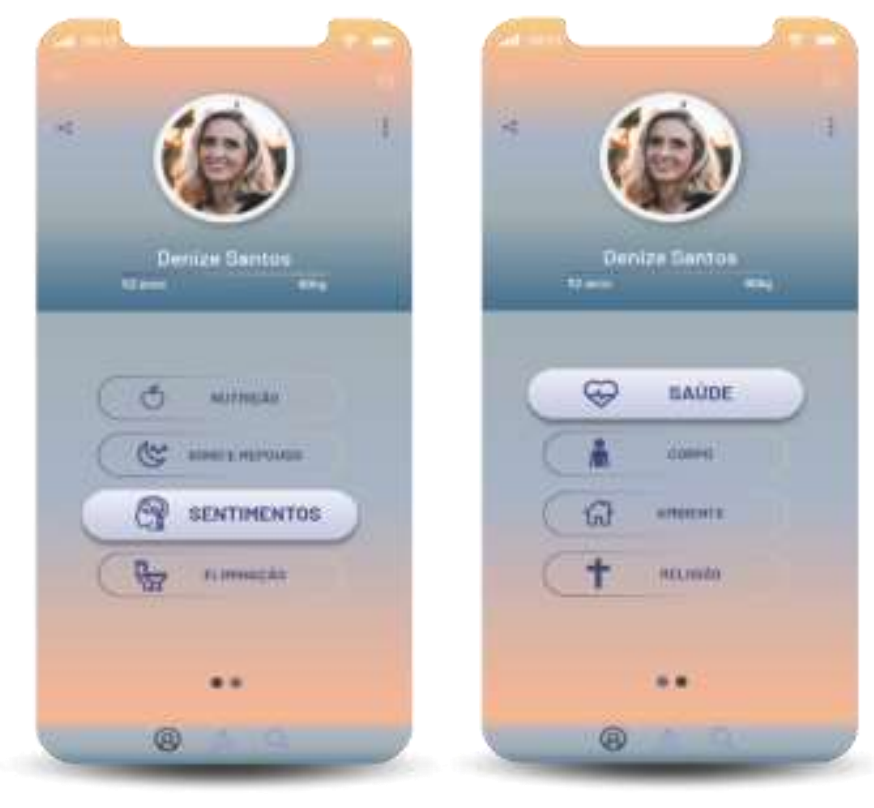

Fonte: Autores.

Os domínios de Nutrição; Sono e Repouso; Eliminação; Mecânica Corporal; Ambiente; Regulação Térmica; Amor; Auto Estima e Necessidades Psicoespirituais, presentes na Teoria de Necessidades Humanas Básicas (Horta,1979), embasaram os requisitos funcionais da tecnologia do protótipo em papel. Contudo, a nomenclatura destes domínios foi adaptada em oito categorias para o protótipo: Nutrição, Sono e repouso, Eliminação; Corpo; Saúde; Ambiente; Religião (correspondente ao domínio Necessidades Psicoespirituais) e Sentimentos (correspondente aos domínios “Amor” e Autoestima"), para facilitar a compreensão no cotidiano dos usuários.

A funcionalidade da tecnologia (apresentada por este protótipo) é caracterizada como um aplicativo de galeria de áudios, onde o usuário poderá selecionar uma dessas categorias para expressar uma necessidade específica, ativando-se a emissão de um som pelo seu aparelho celular. Na tela inicial do aplicativo (Figura 1), o usuário encontrará as categorias com o título adaptado para o protótipo e, ao selecionar uma destas, encontrará subcategorias correspondentes à necessidade que desejará comunicar (Figura 2 e Figura 3), por meio de um clique, o aparelho celular emitirá um som, previamente gravado. Ou seja, caso o usuário queira expressar algo sobre a Necessidade Humana Básica (NHB) de Nutrição, este deverá selecionar no aplicativo, a categoria Nutrição, e posteriormente, caso queira relatar inapetência, selecionará a subcategoria "sem apetite". Deste modo, poderá expressar sua necessidade, clicando o seu aparelho celular, que estará programado para emitir um áudio de uma mensagem, que deixará o receptor e o usuário ciente da informação emitida. 
Figura 2 - Representação das subcategorias do App 1. Belém, PA, Brasil, 2021.

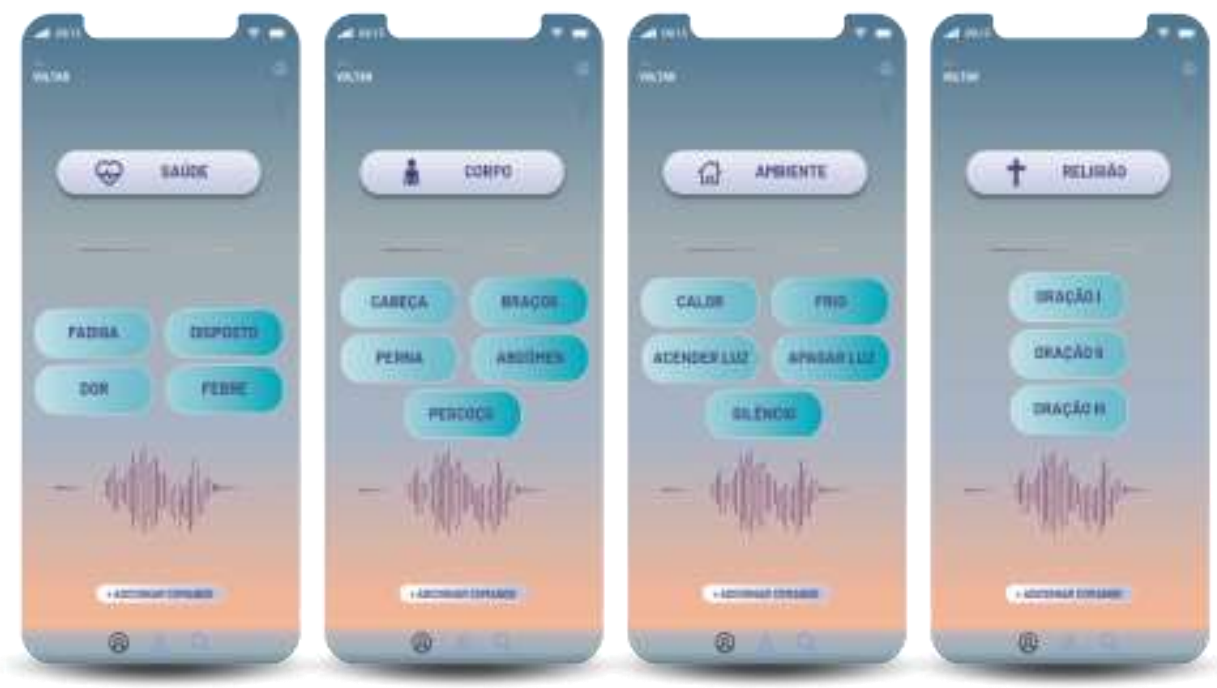

Fonte: Autores.

Figura 3 - Representação das subcategorias do App 2. Belém, PA, Brasil, 2021.
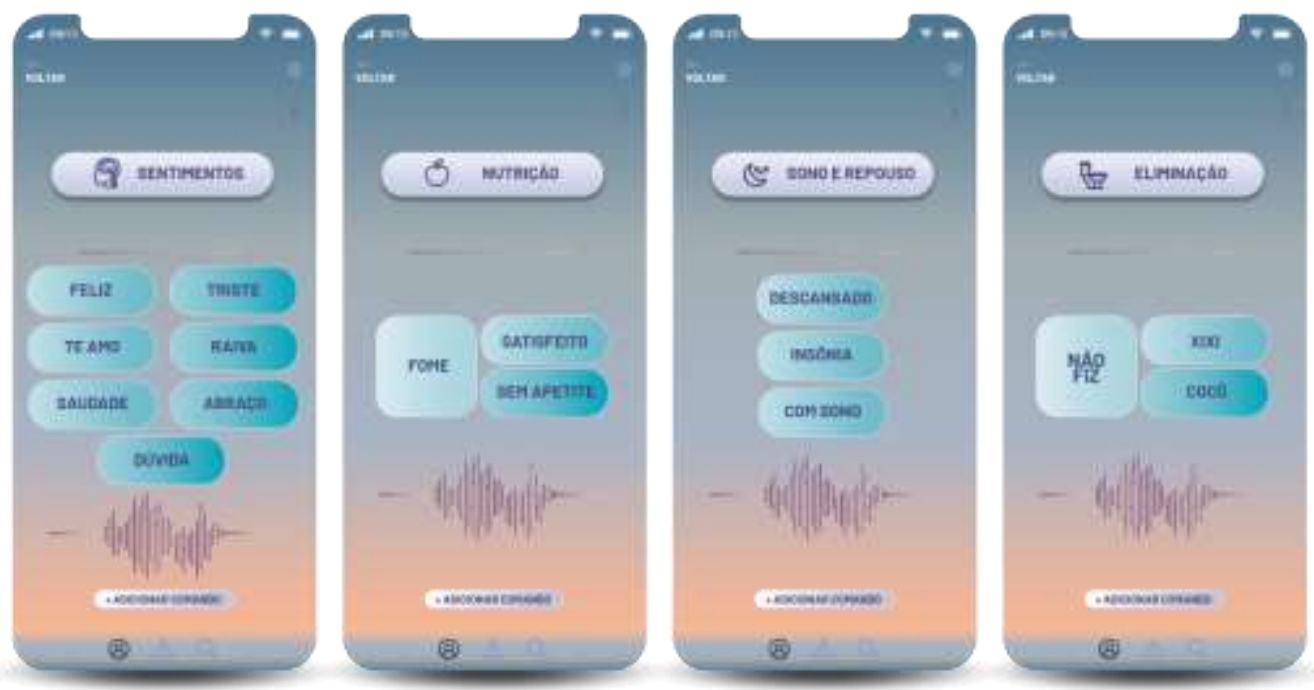

Fonte: Autores.

$\mathrm{Na}$ análise sobre as opções economicamente viáveis para a posterior construção do aplicativo deste protótipo, citam-se as plataformas de desenvolvimento de aplicativos online, que permitem que toda pessoa seja capaz de programar, desenvolver e publicar a sua própria tecnologia. Dentre estas escolheu-se a plataforma FabApp, utilizada em estudos científicos, por grandes e pequenas empresas e, até mesmo, por órgãos públicos, que possibilita a criação de galerias de áudio, oferecimento de profissionais da tecnologia da computação para auxiliar a sua programação. Portanto, estes fatores foram decisivos para a escolha desta plataforma, para a viabilização estrutural e econômica da ideia de um aplicativo sonoro. 


\section{Discussão}

Com o avanço do estadiamento da doença oncológica, bem como diante do tratamento cirúrgico e/ou clínico ao qual são submetidos, a capacidade de comunicação pode diminuir o que pode gerar tensão, ansiedade e frustração no adoecido por câncer de laringe e seus familiares (Cabral, 2017). Nesse contexto, é de fundamental importância que, inicialmente, a assistência de enfermagem à pessoa com traqueostomia por câncer de laringe esteja embasada diante dos diagnósticos de: Conhecimento Deficiente (Herdman \& Kamitsuru, 2018), pois o enfermeiro ensina a pessoa com câncer de laringe e seu familiar cuidador sobre o estado de saúde e as repercussões do tratamento cirúrgico para a vida diária, assim como na diminuição da capacidade de comunicação verbal; Comunicação Verbal Prejudicada (Herdman \& Kamitsuru, 2018), pelo qual o enfermeiro auxilia no desenvolvimento das habilidades para o gerenciamento da afonia e da traqueostomia com as alternativas de comunicação.

Em se tratando das alternativas de comunicação, Klaske et al. (2017) apontam que quando os participantes tiveram que julgar as formas de reabilitação vocal, nenhuma diferença foi encontrada em aceitabilidade entre os métodos. Quanto a qualidade de voz, houve uma melhor qualidade de voz para FTE em comparação com FE, no entanto embora a FTE tenha sido associada ao mais alto nível de inteligibilidade dos alto-falantes, em comparação com as outras abordagens, a qualidade do som ainda é aproximada, baixa afinação e pouco clara em comparação à produção normal de voz.

A eletrolaringe ou laringe eletrônica, é um equipamento eletrônico movido a bateria recarregável, no qual o paciente pressiona o equipamento na região submandibular ou na porção mediana do pescoço, onde é emitida uma vibração sonora contínua, que é transmitida ao ressonador buconasofaríngeo, e pelos órgãos articuladores, como lábios, língua e dentes, dando origem a fala por eletrolaringe (FEL), a qual no estudo de Klaske et al. (2017) foi classificada como uma forma de comunicação alternativa que proporciona uma melhor qualidade de voz e menor riscos ao paciente por se tratar de um recurso tecnológico não invasivo. A portaria $\mathrm{n}^{\circ}$ 39, de 11 de setembro de 2018 e o Projeto de Lei 4324/20 tornam obrigatória a distribuição e incorporação da laringe eletrônica no Sistema Único de Saúde brasileiro, no entanto algumas barreiras ainda são encontradas em relação a aquisição da laringe eletrônica, em virtude do elevado custo e da disponibilidade do equipamento no mercado.

A incorporação de tecnologias no sistema público de saúde e o crescente movimento diante do desenvolvimento e incorporação de recursos de tecnologia móvel na saúde, estão embasados pelos programas da Organização Mundial da Saúde (OMS) M-Health e E-Health, os quais dispõe da implementação do uso das tecnologias da informação e comunicação (TIC's) por meio de dispositivos móveis (como telefones celulares, tablets, dentre outros) em apoio à saúde e nos campos relacionados à saúde, incluindo-se serviços de saúde, vigilância em saúde, literatura em saúde e educação em saúde, conhecimento e pesquisa.

Quanto a categoria profissional, o estudo de Ventola (2014) aponta que os médicos são os profissionais que mais utilizam e desenvolvem recursos de tecnologia móvel para auxiliar na assistência e no ensino. Historicamente, a utilização de recursos de tecnologia da informação é uma realidade do cotidiano do profissional de enfermagem, no entanto ainda é vista como um desafio à prática dos enfermeiros (Marin \& Cunha, 2006). No que diz respeito a utilização e desenvolvimento de recursos de tecnologia móvel o estudo de Silva et al. (2018) aponta que a temática é recente e desenvolvida em países onde há mais recursos tecnológicos. Para os profissionais, as possibilidades de criação de tecnologia móvel vão desde o registro de dados do paciente, meio de informação sobre doenças, atividades gerenciais e administrativas, dentre outras. Identificou-se também uma necessidade de novos estudos sobre tecnologias móveis na área da Enfermagem, a fim de que possam preencher as lacunas atualmente presentes e contribuir para prática de enfermagem ágil, sistematizada e segura.

A pesquisa nacional por amostra de domicílios contínua (tecnologia da informação e comunicação), realizada pelo Instituto Brasileiro de Geografia e Estatística (2018), evidenciou que 79,3\% dos brasileiros possuem aparelhos celulares para o 
uso pessoal, e que 98,1\% desses usuários tem acesso à internet por meio dos celulares, em relação aos entrevistados que declararam não possuir celular para o uso pessoal (20,7\%), apontando como justificativa o alto custo para a aquisição do recurso tecnológico, falta de interesse e/ ou falta de habilidade para manuseio do aparelho.

Pensando na perspectiva da crescente utilização de recursos de tecnologia da informação para o uso pessoal e para a assistência em saúde, e principalmente no reconhecimento da importância da inserção e do acompanhamento das novas formas de produzir o Cuidado e a Ciência da Enfermagem frente a revolução técnico-cientifica informacional, teve-se como proposta o desenvolvimento de um protótipo de uma tecnologia de comunicação alternativa, o qual visasse atender às necessidades da pessoa com traqueostomia por câncer de laringe quanto a expressão de suas necessidades fisiológicas e emocionais.

Nesse contexto, o protótipo apresentado caracteriza-se como um produto de inovação tecnológica móvel, que constitui a primeira comunicação alternativa móvel desenvolvida no Brasil, e fundamentado na Teoria de Necessidades Humanas Básicas. Assim como também, trata-se de uma intervenção de enfermagem ao paciente com traqueostomia por câncer de laringe, mas poderá beneficiar outros pacientes com o Diagnóstico de Enfermagem "Comunicação Prejudicada", cuja utilização poderá ser extrapolada para o contexto domiciliário do binômio adoecido-familiar cuidador.

A acessibilidade às alternativas de comunicação como o desenvolvimento da voz esofágica e aquisição da laringe eletrônica, ainda é um desafio na realidade destas pessoas e este protótipo contribuirá na melhoria das relações interpessoais com os familiares e com a sociedade, auxiliando na solução de adversidades, como uma ferramenta importante para o ajustamento social e reabilitação destas pessoas, assim como demonstrado nos estudos de Brunner (2016) e Brunner (2018) a respeito do satisfação da pessoa com câncer de laringe em relação utilização de tecnologias móveis para a comunicação.

As limitações deste estudo se devem ao fato desta tecnologia de protótipo ainda não ter concluído a fase de manutenção. Contudo, a viabilização das etapas de definição e de desenvolvimento, possibilitaram a proposição e sua fundamentação metodológica, que favorecerão as sugestões de adequações no processo de sua validação que requer o desenvolvimento de aspectos técnicos e de conteúdo do aplicativo de comunicação alternativa, para assegurar a efetividade, viabilidade, confiabilidade e relevância para a reabilitação, cuidado e adaptação da pessoa com traqueostomia por câncer de laringe diante da impossibilidade da produção de voz.

\section{Considerações Finais}

O método de prototipação viabilizou a ideação de uma tecnologia, o aprofundamento teórico nesta metodologia, bem como planejar e discutir minunciosamente cada uma das etapas, confirmando-se a viabilidade do desenvolvimento de um software, ancorado em uma Teoria de Enfermagem, neste caso, um aplicativo móvel como alternativa para a comunicação da pessoa com traqueostomia por câncer de laringe. Posteriormente, será realizado o desenvolvimento e validação da tecnologia deste protótipo por estes usuários, pelos seus familiares cuidadores, e pelos pares, ou seja, enfermeiros com expertise.

A utilização de recursos tecnológicos é uma realidade no cotidiano da profissão de Enfermagem, no entanto, a produção científica sobre a incorporação de novas tecnologias como celulares e tablets no cuidado, assim como do desenvolvimento e uso de softwares de tecnologia móvel ainda são escassas no Brasil, principalmente para pacientes oncológicos. Diante da pressão social da utilização massiva de recursos de mídias móveis (celulares e tablets), esta proposta favorece o ensino, seguimento de pacientes e continuidade da assistência, após a alta hospitalar.

Espera-se que o produto desta pesquisa, bem como os seus passos metodológicos possam embasar a incorporação de conhecimentos da área de Tecnologias da Informação pela enfermagem e pela área da saúde, estimulando a proposição de outros protótipos para o cuidado em Saúde, para implementação na prática clínica assistencial, como resultado do avanço científico em saúde e na Enfermagem, favorecendo a satisfação e qualidade de vida dos usuários, bem como do cuidado prestado pelos enfermeiros e pela equipe multiprofissional. 


\section{Referências}

Agboola, S. O., Bates, D. W., \& Kvedar, J. C. (2016). Digital Health and Patient Safety. JAMA, 315(16), 1697-1698. https://doi.org/10.1001/jama.2016.2402

Brasil. Ministério da Saúde. Secretaria de Ciência, Tecnologia e Insumos Estratégicos. (2018). Portaria $\mathrm{n}^{\circ} 39$, de 11 de setembro de 2018. https://bvsms.saude.gov.br/bvs/saudelegis/sctie/2018/prt0039_12_09_2018.html.

Brasil. Câmara dos Deputados. Projeto de Lei No 4324/2020. (2020). https://www.camara.leg.br/propostas-legislativas/2260853

Brunner, T. H., Kristyn DiFortuna, K. D., Michael LeTang, M. L., Murphy, J., Stemplewicz, K., Magda Kovacs, M. K., DeRosa, A. P., Gibson, D. S., \& Ginex, P. K. (2016). Using Technology to Give Patients a Voice After Surgery for Head and Neck Cancer. Clinical journal of oncology nursing, 20(5), 474476. https://doi.org/10.1188/16.CJON.474-476

Brunner, T. H., DiFortuna, K., LeTang, M., Murphy, J., Stemplewicz, K., Kovacs, M., DeRosa, A. P., Gibson, D. S., \& Ginex, P. K. (2018). Feasibility of an iPad to Facilitate Communication in Postoperative Patients With Head and Neck Cancer. Journal of perianesthesia nursing: official journal of the American Society of PeriAnesthesia Nurses, 33(4), 399-406. https://doi.org/10.1016/j.jopan.2016.10.008

Cabral, G. K. A., Araújo, M. A. M., Leitão, B. F. B., Rodrigues, A. B., \& Gomes, A. M. L. (2017). A comunicação em pacientes oncológicos submetidos à laringectomia total. Revista da SBPH, 20(2), 45-65. http://pepsic.bvsalud.org/pdf/rsbph/v20n2/v20n2a04.pdf

Caldas, A. S. C., Facundes, V. L. D., Melo, T. M. A., Dourado Filho, M. G., Pinheiro Júnior, P. F., \& Silva, H. J. (2011). Alterações e avaliação das funções do olfato e do paladar em laringectomizados totais: revisão sistemática. Jornal da Sociedade Brasileira de Fonoaudiologia, 23(1), 8288. https://doi.org/10.1590/S2179-64912011000100017

Deloitte Touche Tohmatsu Limited. (2016). Mobile Consumer Survey 2016: hábitos dos usuários e tendências para o mercado de telecomunicações. https://www2.deloitte.com/br/pt/pages/technology-media-and-telecommunications/articles/mobile-survey.html..html

FabApp (Fábrica de aplicativos). Site desenvolvedor de aplicativos. https://fabricadeaplicativos.com.br/

Fogle, P. T. (2013). Essentials of communication sciences \& disorders. Clifton Park: Delmar, Cengage Learning.

Galbiatti, A. L., Padovani-Junior, J. A., Maníglia, J. V., Rodrigues, C. D., Pavarino, E. C., \& Goloni-Bertollo, E. M. (2013). Head and neck cancer: causes, prevention and treatment. Braz J Otorhinolaryngol, 79(2), 239-247. https://doi.org/10.5935/1808-8694.20130041

Gil, A. C. (2010). Como elaborar projetos de pesquisa. (5a ed.), Atlas.

Herdman,T. H., \& Kamitsuru, S. (2018). Diagnósticos de enfermagem da NANDA: definições e classificação 2018-2020. Artmed.

Horta, W. A. (1979). Processo de enfermagem. EPU.

Instituto Brasileiro de Geografia e Estatística (IBGE). (2018). Pesquisa Nacional por amostra de domicílios contínua (tecnologia da informação e comunicação) - Acesso à internet, à televisão e posse de telefone móvel celular para uso pessoal https://ftp.ibge.gov.br/Trabalho_e_Rendimento/Pesquisa_Nacional_por_Amostra_de_Domicilios_continua/Anual/Acesso_Internet_Televisao_e_Posse_Telefo ne_Movel_2018/Analise_dos_resultados_TIC_2018.pdf

Juliani, C. M. C. M., Silva, M. C., \& Bueno, G. H. (2014). Avanços da informática em enfermagem no Brasil: revisão integrativa. J Health Inform, 6(4), 161165. http://jhi-sbis.saude.ws/ojs-jhi/index.php/jhi-sbis/article/view/322

Klaske, E., Molen, L., Hilgers, F., Bhairosing, P. A., \& Brekel, M. (2018). Objective and subjective voice outcomes after total laryngectomy: a systematic review. European archives of oto-rhino-laryngology, 275(1), 11-26. https://doi.org/10.1007/s00405-017-4790-6

Marin, H. F., \& Cunha, I. C. K. O. (2006). Perspectivas atuais da Informática em Enfermagem. Revista Brasileira de Enfermagem, 59(3), 354-357. https://doi.org/10.1590/S0034-71672006000300019

Melo Filho, M. R., Rocha, B. A., Pires, M. B. O., Fonseca, E. S., Freitas, E. M., Martelli Junior, H., \& Santos, F. B. G. (2013). Quality of life of patients with head and neck cancer. Braz J Otorhinolaryngol, 79(1):82-8. https://doi.org/10.5935/1808-8694.20130014

Neves, W. F. S., \& Santana, M. E. (2020). A Prototipação de uma Tecnologia de Comunicação Alternativa Para o Adoecido de Câncer de Laringe (Projeto de Pesquisa). Universidade Federal do Pará, Belém, PA, Brasil.

Othero, M. \& Costa, D. (2007). Propostas desenvolvidas em cuidados paliativos em um Hospital Amparador-Terapia Ocupacional e Psicologia. Revista Prática Hospitalar, 9(52), 157-160.

Pelosi, M. B. (2011). Projeto de Pesquisa e Extensão: Implementação da Comunicação Alternativa para os Pacientes com Dificuldade de Fala no Hospital Universitário Clementino Fraga Filho. Rio de Janeiro. https://drive.google.com/viewerng/viewer? a=v\&pid=sites\&srcid=ZGVmYXVsdGRvbWFpbn x0ZWNub2xvZ2lhYXNzaXN0aXZhdWZyanxneDo1MTcyNDExNzBkY2Y1NjZl

Pressman, R. S. (2006). Engenharia de Software. (6a ed.), McGraw/Hill/Nacional.

Silva, A. M. A., Mascarenhas, V. H. A., Araújo, S. M., Machado, R. S., Santos, A. M. R. \& Andrade, E. M. L. R. (2018). Tecnologias móveis na área de Enfermagem. Revista Brasileira de Enfermagem, 71(5), 2570-2578. https://doi.org/10.1590/0034-7167-2017-0513

Silveira, A., Gonçalves, J., Siqueira, T., Ribeiro, C., Lopes, C. \& Monteiro, E. et al. (2012). Oncologia de cabeça e pescoço: enquadramento epidemiológico e clínico na avaliação da qualidade de vida relacionada com a saúde. Rev bras epidemiol, 15(1), 38-48. https://www.scielosp.org/article/rbepid/2012.v15n1/38$48 /$

Sommerville, I. (2011). Engenharia de Software. (9a ed.) Pearson Education do Brasil. 
Research, Society and Development, v. 10, n. 4, e26410413990, 2021

(CC BY 4.0) | ISSN 2525-3409 | DOI: http://dx.doi.org/10.33448/rsd-v10i4.13990

Ventola C. L. (2014). Mobile devices and apps for health care professionals: uses and benefits. $P$ \& $T$ : a peer-reviewed journal for formulary management, 39(5), 356-364. https://www.ncbi.nlm.nih.gov/pmc/articles/PMC4029126/

World Health Organization. (2011). mHealth: new horizons for health through mobile technologies. Global Observatory for eHealth Series 3. http://www.who.int/goe/publications/goe_mhealth_web.pdf 